\title{
Experience of a single-center in coronary computed tomography angiography
}

\author{
Željko Madžar*, Ana Lanča, Vedran Buršić, Dragan Javoran, Vladimir Peša, \\ Vesna Pehar Pejčinović, Marko Boban, Viktor Peršić \\ Thalassotherapia Opatija - Clinic for treatment, rehabilitation and prevention of cardiovascular \\ disease, Opatija, Croatia
}

Purpose: Coronary computed tomography angiography with calcium scoring measured with Agatston score index provides a diagnosis of coronary atherosclerosis in the patients with subclinical coronary plaque. To evaluate the diagnostic accuracy of Agatston score in comparison with the conventionally used Framingham risk score (FRS) and blood biomarkers in predicting existence of coronary artery disease (CAD) and significant coronary stenosis $>50 \%$.

Patients and Methods: This retrospective single-center study is evaluating the role of Agatstone score in the prognosis of CAD in patients with previously unknown CAD. 2,597 patients underwent coronary multidetector computed tomography (MDCT) and obtained Agatston score in our Center from June 2012 to April 2014. Scanning was done with dualsource MDCT equipped with two 128-detector row units using the ECG-gating protocol. The mean amount of radiation that was obtained was $6.8 \pm 4.5 \mathrm{mSv}$. The exclusion criteria included Agatston score above 800, atrial fibrillation, pre-existing kidney failure (eGFR $<50 \mathrm{~mL} / \mathrm{min} / 1.73 \mathrm{~m}^{2}$ ), as well as the incomplete data. All of the patients with heart rate higher than $80 / \mathrm{min}$ received beta blocker. The mean

Received: $19^{\text {th }}$ Apr 2014

*Address for correspondence: Thalassotherapia Opatija, Maršala Tita 188/1, HR51410 Opatija, Croatia.

Phone: +385-91-5537229

E-mail: zmadzar@yahoo.com heart rate during scanning was $68 \pm 9 / \mathrm{min}$, and the patient's body mass index was $28 \pm 5,1$. The participants were divided into two groups based on coronary artery stenosis, significant $>50 \%$ or less than $50 \%$. The frequency of demographic and clinical characteristics, blood biomarkers and risk factors were tested between the groups by using chi-squared test and Student T-test as appropriate. To test for factors associated with predicting coronary artery stenosis, likelihood ratio, and receiver operating characteristics analyses were used. Relative risk was calculated in the context of developing coronary artery stenosis greater than $50 \%$ in the participants with Agatston score above 100.

Results: Out of 2,597 patients participating, 361 patients (mean age $63 \pm 8 ; 227$ (63\%) of females) with complete data were included in this study. Significant coronary artery stenosis was recorded in $84(23 \%)$ of patients. Mean FRS values were $20 \pm 13$. Agatstone score (Se $85 \%$, Sp 95\%, NPV 95\%, PPV 85\%, AUC 0.99, 95\% Cl 0.968 to 1.000 , $\mathrm{p}<0.0001)$.

Conclusion: Among all considered demographic and clinical characteristics, Agatstone score was the most accurate predictor for developing of CAD and significant coronary stenosis.

KEYWORDS: computed tomography, coronary angiography, calcium score index, predictors, coronary artery disease.

CITATION: Cardiol Croat. 2014;9(5-6):193. 